\title{
Calentamiento global y la fisiología de ectotermos: el caso de tres lacertilios mexicanos
}

\section{Global warming and the ectothermic-animal physiology: The case of three Mexican lacertilians}

\author{
Victor Fajardo \\ Instituto para la Conservación de la Cordillera Neovolcánica ante el \\ Cambio Climático, México \\ fajardo.vic@gmail.com \\ (D) http://orcid.org/0000-0001-9253-0145
}

Recepción: 9 de julio de 2018

Aprobación: 17 de diciembre de 2019

Marycarmen Burguete

Instituto para la Conservación de la Cordillera Neovolcánica ante el Cambio Climático, México

mvzmburguete@gmail.com

(D) http://orcid.org/0000-0001-7962-2643

Juan Carlos González-Morales

Instituto para la Conservación de la Cordillera Neovolcánica ante el Cambio Climático, México

juan.gonmor@gmail.com

(D) http://orcid.org/0000-0003-2759-0357

\section{RESumen}

Los hallazgos recientes muestran que el calentamiento global puede causar cambios en la distribución espacial de diversas especies de reptiles e incluso llevar a su extinción. Se ha propuesto teóricamente que los lacertilios podrían migrar en un gradiente altitudinal; sin embargo, en estas propuestas no se ha considerado a la hipoxia (escasez de oxígeno) como un factor limitante de la migración. De acuerdo con lo anterior, en este artículo se discuten las posibles características morfofisiologicas que podrían permitir a los ectotermos migrar sobre un gradiente altitudinal aun con los efectos negativos de la hipoxia. Es fundamental estudiar a profundidad la vulnerabilidad de los ectotermos ante el calentamiento global tomando en cuenta no solo su biología térmica, sino también su fisiología.

Palabras clave: calentamiento global, lagartijas, ectotermos, hipóxia, biogeografía.

\begin{abstract}
Global warming has a direct impact on the geographic distribution and abundance of reptile species, and it can even lead to their extinction. Theoretical models project that lacertilians could migrate following an altitudinal gradient, however, this prediction has not been considered the lack of oxygen or hypoxia as a constraint factor of migration. We discuss possible morphophysiological traits that could allow ectotherms to migrate over an altitudinal gradient even with the negative effects of hypoxia. It is fundamental that climate change research considers the vulnerability of ectotherms facing global warming, not only in terms of their thermal biology, but also considering their physiology.
\end{abstract}

KEYWORDs: global warming, lizards, ectotherms, hypoxia, biogeography.

\section{Calentamiento global y ectotermos}

Este artículo se encuentra conformado por tres manuscritos que publicaron los autores en revistas internacionales y tiene como finalidad de divulgar dichos estudios entre los lectores de CIENCIA ergo-sum. 
Los datos de la NOAA (National Oceanic and Atmospheric Administration) han servido de base para innumerables estudios científicos que demuestran un incremento gradual de la temperatura media del planeta (Petit et al., 2009; Barry y Chorley, 2003; Brohan et al., 2006) (gráfica 1). Este aumento es de casi $0.5^{\circ} \mathrm{C}$ si tomamos como base la temperatura media registrada entre 1961 y 1990 , y de casi $1{ }^{\circ} \mathrm{C}$ si la comparamos con la segunda mitad del siglo XIX, esto es, entre 1850 y 1900 (Caballero et al., 2007). El calentamiento global ha ido de la mano con una tendencia hacia un incremento en el $\mathrm{CO}_{2}$ atmosférico, lo que es un fuerte indicador de que una de las principales causas de la tendencia hacia el calentamiento es la intensificación del efecto invernadero (Caballero et al., 2007). De allí que con frecuencia se usen indistintamente ambos términos, pero mientras uno describe el fenómeno del incremento de temperatura, el otro se refiere al mecanismo que lo causa (Caballero et al., 2007).

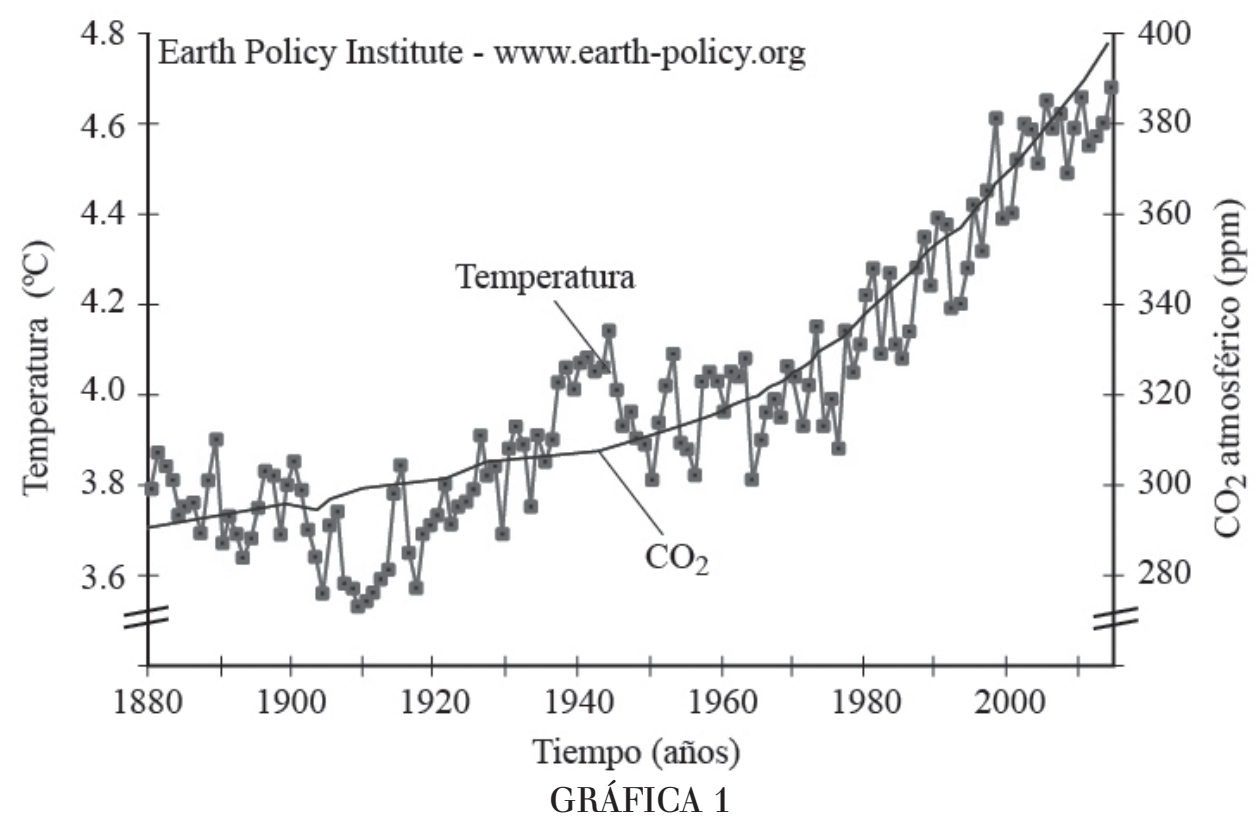

Temperatura y $\mathrm{C}_{2}$ en el transcurso de los años Fuente: elaboración propia basada en los datos de la NOAA. Nota: nótese el incremento en ambos parámetros.

El calentamiento global es también detectable a nivel local. En la gráfica 2 se muestran los datos obtenidos de la estación meteorológica de Tenango del Valle, Estado de México, donde se evidencia que a partir de la década del 2000 se incrementa constantemente la temperatura del aire. A pesar de que se observan temperaturas bajas en las décadas de los ochenta y noventa, la tendencia a la elevación de las temperaturas ambientales es irrefutable, por lo que hay un claro consenso científico que alerta de las consecuencias de este incremento de temperatura. Por ejemplo, el último informe emitido por el Panel Intergubernamental sobre el Cambio Climático (IPCC por sus siglas en inglés) de la ONU, el cual está conformado por miles de científicos de todo el mundo, nos informa que estamos próximos a pasar el umbral crucial de $1.5^{\circ} \mathrm{C}$ por encima de los niveles de temperatura preindustriales, lo que cambiaría ecosistemas desertificándolos, aumentarían las sequías extremas y lo incendios naturales, así como las inundaciones (IPCC, 2018).

Aunado a lo anterior, diversos estudios han especulado que el calentamiento global causará la extinción de especies, así como cambios en su distribución espacial, aunque existen pocos datos que validen dichas predicciones (Sinervo et al., 2010, 2018). Particularmente, para el caso de los reptiles como los lacertilios, el calentamiento global es una fuerza selectiva muy poderosa, pues desde el punto vista metabólico los vertebrados pueden ser clasificados de acuerdo con la forma en que producen y mantienen su temperatura corporal en dos grandes grupos: a) ectotermos y $b$ ) endotermos. Ambos grupos presentan tres grandes diferencias entre 
sí: a) Los ectotermos (como los reptiles, y por ende los lacertilios) adquieren su calor corporal por medio de la exposición a fuentes calóricas del ambiente; por el contrario, los endotermos producen su calor corporal mediante procesos metabólicos que implican transformar el alimento ingerido en calor, lo que implica costos tróficos más altos. $b$ ) Cuando la temperatura ambiental disminuye en los ectotermos, al mismo tiempo lo hace su temperatura corporal y con ello su metabolismo. Por ejemplo, durante la noche los ectotermos utilizan sólo una tercera parte de la energía que utilizan durante el día; sin embargo, para la mayoría de los endotermos la disminución de la temperatura en la noche juega un papel inverso debido a que están obligados a mantener una temperatura corporal constante, por lo que se ven en la necesidad de aumentar su producción de calor. c) La necesidad de mantener la temperatura corporal hace que un organismo endotermo invierta más tiempo forrajeando y de esta manera consuma suficiente alimento para mantener su temperatura corporal; en su caso los ectotermos invierten una menor cantidad de tiempo y necesitan menor cantidad de alimento, ya que la temperatura la modulan en función del ambiente, por lo que ese tiempo extra lo invierten en reproducción y fertilidad (Pough, 1980; Pough et al., 2001).

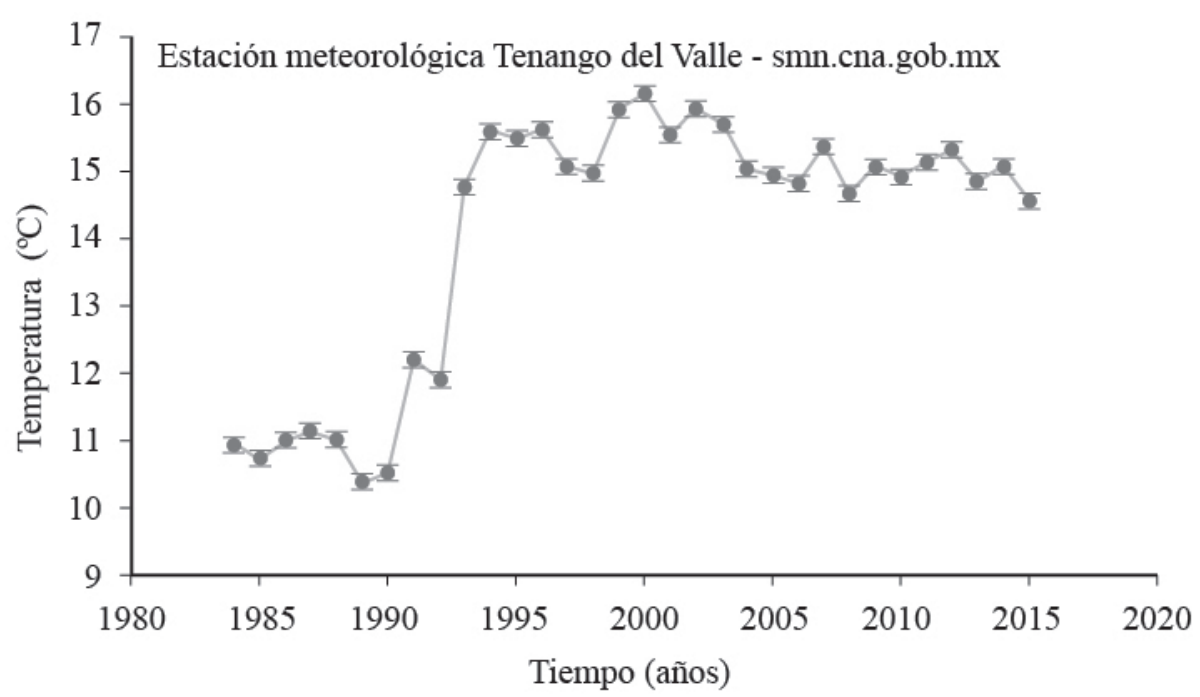

GRÁFICA 2

Temperatura en el transcurso de los años.

Fuente: elaboración propia basada en los datos de la Estación Metereológica de Tenango del Valle. Nota: nótese el incremento.

A pesar de que los organismos ectotermos presentan varias ventajas sobre los endotermos, existen importantes costos asociados con la termorregulación ectotérmica; la imitación impuesta por las temperaturas ambientales a la posibilidad de aumentar su distribución espacial (en latitud y/o altitud) se encuentra entre las más importantes, que es contrario a lo acontecido con la regulación interna de los endotermos que les permite distribuirse casi ilimitadamente en el espacio, lo cual explica la existencia de endotermos en ecosistemas donde la temperatura ambiental suele ser baja durante todo el año, como en las zonas polares (Pough, 1980; Pough et al., 2001).

Con esta información, diversos grupos de herpetólogos han comenzado a analizar el efecto del calentamiento global sobre especies de reptiles (Sinervo et al., 2010; 2018) y predecir cómo estas pueden hacerle frente. Una idea que se ha manejado en la literatura es que los reptiles, incluyendo los lacertilios, podrían migrar sobre un gradiente altitudinal, como es el caso de las montañas, para buscar sitios térmicamente adecuados. Sin embargo, se sabe que los ambientes con mayor altitud, además de temperaturas, bajas presentan menor cantidad de oxígeno disponible, fenómeno llamado hipoxia. Se ha sugerido que la hipoxia no es el único factor que limita la distribución de los reptiles en ambientes de alta altitud, por lo que se han realizado 
estudios tratando de entender qué cambios presentan estos organismos para sobrevivir en estos ambientes (Snyder y Weathers, 1997; Sinervo et al., 2010, 2018).

Por otra parte, puesto que el oxígeno es esencial para mantener la vida tanto de animales ectotermos como endotermos, ambos deben presentar una serie de mecanismos que les permitan aclimatarse o adaptarse a ambientes con hipoxia. En ectotermos estas adaptaciones incluyen una gran variedad de cambios morfofisiológicos en el sistema cardiovascular y respiratorio (Bouverot, 1985). Por ejemplo, una posible estrategia consiste en aumentar el transporte de oxígeno en la sangre mediante el aumento en el número de eritrocitos, hematocrito y concentraciones de hemoglobina y modificaciones en el área de los eritrocitos; aunque no en todos los ectotermos se presentan estos cambios, sí se han sido descrito en peces, ranas y lagartijas (Ruiz et al., 1983, 1989; González-Morales et al., 2015). Como complemento a los cambios morfofisiológicos se encuentran los del metabolismo, ya que a menor oxígeno el metabolismo en animales ectotérmicos tiende a suprimirse (Wheathers y Withe, 1972).

\section{Hipoxia Y LACERTILIOS}

Ante un escenario de elevación de temperaturas asociadas al calentamiento global, las especies ectotérmicas tendrían la opción de migrar de su lugar de residencia a lugares menos cálidos, pero esto sólo será posible en aquellas especies que puedan responder morfofisiológicamente o metabólicamente a la hipoxia (Storz y Moriyama, 2008; González-Morales et al. 2015, 2017). Los mecanismos de plasticidad o flexibilidad fenotípica para hacer frente a la hipoxia en una migración altitudinal como respuesta al calentamiento global se han analizado a través del estudio de diversos vertebrados residentes en altas y bajas elevaciones. En ellos, los rasgos hemáticos, como el conteo de eritrocitos y porcentaje de hematocrito y concentración de hemoglobina, tienden a incrementar o disminuir. Dichos ajustes hemáticos no son uniformes en todas las clases de vertebrados; por ejemplo, en mamíferos y aves (salvo pocas excepciones) la respuesta a la hipoxia consiste en aumentar los valores hemáticos, un fenómeno denominado respuesta hemática. En anfibios la respuesta es inversa: varios valores como el conteo eritrocitario aumentan, pero su tamaño disminuye. En el caso de los reptiles es difícil establecer un patrón claro, ya que los datos disponibles no son suficientes para establecer una idea concisa; por ejemplo, en nuestro laboratorio hemos descrito que algunas lagartijas del género Sceloporus presentan respuesta hemática a la hipoxia (González-Morales et al., 2015, 2017,2019), pero esta respuesta no se observa en individuos de Heloderma horridum (Guadarrama et al., 2019).

En México se han estudiado a detalle tres lacertilios: Sceloporus torquatus (González-Morales et al., 2015), Sceloporus grammicus (González-Morales et al., 2017) y Heloderma horridum (Guadarrama et al., 2019). Sceloporus torquatus, cuyo nombre común es lagartija de collar o chintete, es una lagartija saxícola (Feria-Ortíz et al., 2001) que se distribuye en el centro de México. De acuerdo con la NOM-059-ECOL-2010, es una especie endémica de México y no está sujeta a ningún tipo de protección especial (Flores-Villela y Gerez, 1994; Uribe-Peña et al., 1999; Méndez de la Cruz y Gutiérrez-Mayen, 1991). Presenta además un tamaño o longitud del hocico a la cola de entre 15 y 20 centímetros y cuando los machos son adultos muestran coloración azul en la gula y en los parches laterales, lo que la hace una lagartija muy llamativa para su extracción ilegal y venta como mascota. Se ha estudiado porque tiene un amplio rango de distribución altitudinal, pues habita desde los 1450 hasta los $3000 \mathrm{~m}$ de altitud y es abundante en zonas moderadamente perturbadas. Esta lagartija despliega respuesta hemática a la hipoxia: incrementa sus valores sanguíneos y con ello su capacidad para transportar oxígeno de los pulmones a los tejidos (González-Morales et al., 2015) (gráfica 3, figura 1). Wheathers y White (1972) fueron los primeros en mostrar que un ectotermo puede responder a la hipoxia y con este conocimiento se hizo factible predecir una migración altitudinal de las lagartijas en el futuro. Sin embargo, aún es necesario entender si la biología térmica de estos organismos le podría permitir dicha migración. 


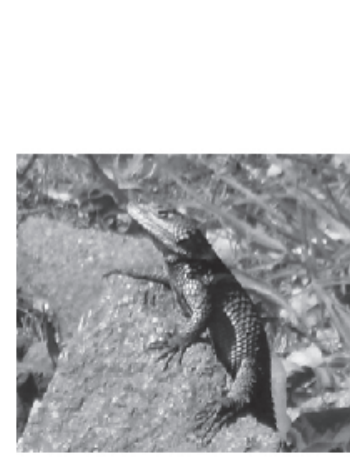

Figura 1

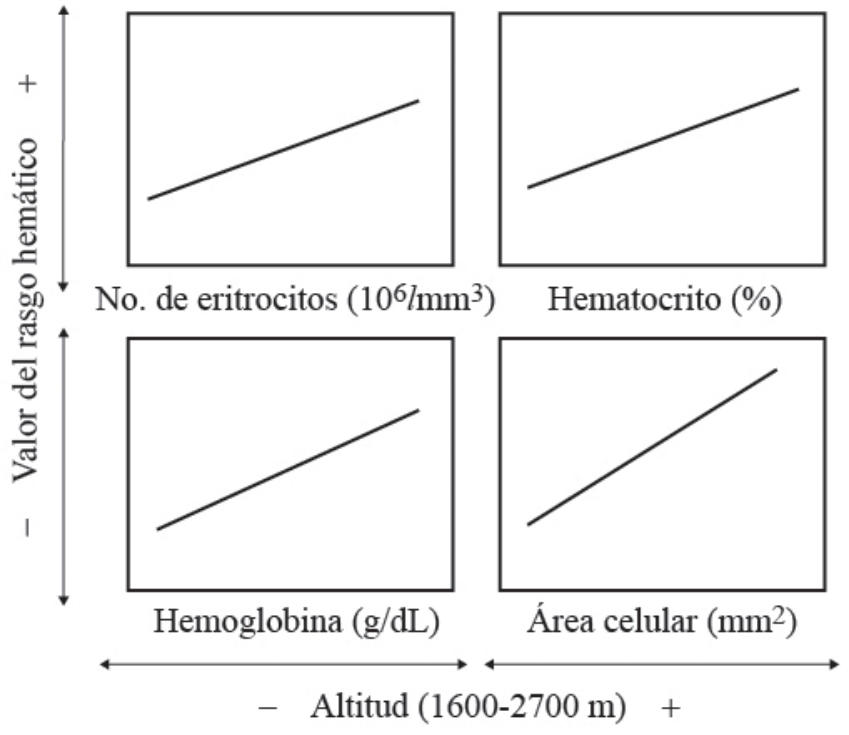

GRÁFICA 3

Valores hemáticos observados en Sceloporus torquatus

Fuente: elaboración propia basada en los datos de González-Morales et al., 2015.

Nota: nótese el incremento lineal que muestra que a mayor altitud mayor rasgo hemático.

Se han establecido dos grandes vertientes de predicción en cuanto a la biología térmica de ectotérmos en ambientes heterogéneos como lo son los gradientes de altitud. a) La primera, propuesta por Adolph y Porter (1993), establece que los rasgos térmicos pueden ser adaptativos al ambiente, es decir, que se pueden modificar por efecto de la estacionalidad, latitud y altitud. La temperatura de selección o la temperatura elegida por los animales en condiciones de laboratorio es alta en primavera y baja en verano con la finalidad de minimizar los costos de termorregulación (Ortega et al., 2016), es el caso de la lagartija Iberalocerta galani. b) La segunda menciona que la biología térmica es conservativa, es decir, que no se modifica por efecto de la estación, latitud y altitud, por lo que ajustes conductuales son suficientes para mitigar los cambios ambientales (Crowley, 1985); por ejemplo, organismos de Sceloporus undulatus muestran temperaturas de selección similares en dos poblaciones de diferente altitud (Crowley, 1985).

Para el género Sceloporus los datos actuales se ajustan de mejor manera a la visión estática, ya que la temperatura corporal y de actividad no se modifican por la altitud y latitud (Andrews, 1998). Esto ha llevado a la idea de que mantener este tipo de termorregulación en ambientes térmicamente desfavorables representa un costo bastante alto, por lo que las lagartijas que habitan en rangos altitudinales extremos deben de presentar una serie de compensaciones a diversos niveles de organización biológicos para sobrevivir y reproducirse en esos lugares, cambios que a la fecha se mantienen desconocidos (Lemos-Espinal y Ballinger, 1995).

Sceloporus torquatus, en su caso, puede ser considerada como una especie euritérmica o que tiene un amplio rango de temperaturas de selección, que en el laboratorio van desde los 22.4 a los $33.7^{\circ} \mathrm{C}$ (Rivera-Rea et al., 2018), por lo que podríamos considerar con estos estudios que $S$. torquatus puede migrar en un gradiente altitudinal y evitar los efectos del calentamiento global. Por otra parte, realizamos otro estudio utilizando una de mayor altitud, Sceloporus grammicus, que es una lagartija arborícola que se distribuye en el centro de México. Su nombre común es lagartija del mezquite y habita desde los $450 \mathrm{~m}$ hasta los $4600 \mathrm{~m}$ de altitud; de acuerdo con la NOM-059-ECOL-2010, es una especie que no está sujeta a ningún tipo de protección especial (Flores-Villela y Gerez, 1994; Uribe-Peña et al., 1999; Méndez de la Cruz y Gutiérrez-Mayen, 1991). Esta lagartija presenta respuesta hemática e incrementa sus valores sanguíneos hasta los $3600 \mathrm{~m}$ de altura; sin embargo, las poblaciones a los $4400 \mathrm{~m}$ presentan valores similares a poblaciones relativamente bajas 
(González-Morales et al., 2017) (gráfica 4, figura 2). Este fue el primer estudio que demostró que hay más de una manera para responder a la hipoxia, dado que existen poblaciones por encima de los $4000 \mathrm{~m}$, pero no muestran incrementos en sus valores hematológicos, adecuaciones morfofisiológicas no entendidas hasta el momento (González-Morales et al., 2017).

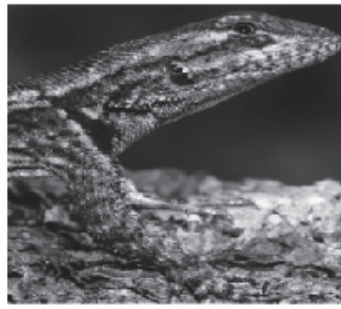

Figura 2
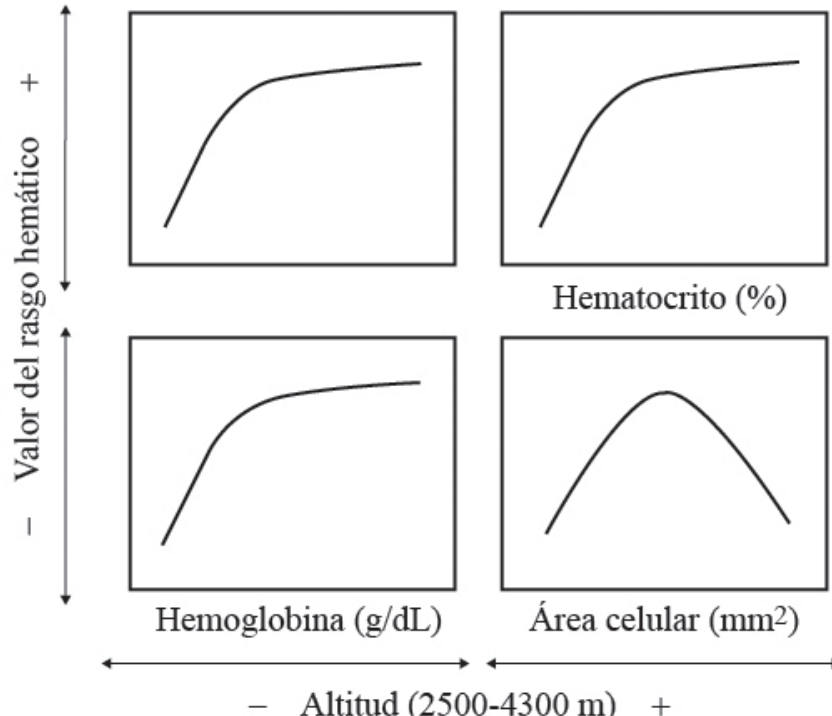

Hematocrito (\%)

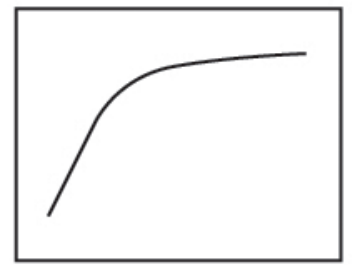

Hemoglobina $(\mathrm{g} / \mathrm{dL})$

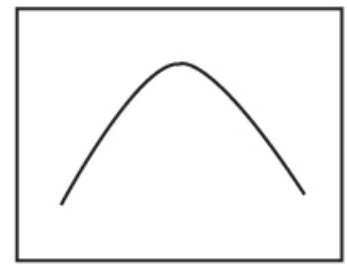

Área celular $\left(\mathrm{mm}^{2}\right)$

- Altitud (2500-4300 m) +

GRÁFICA 4

Valores hemáticos observados en Sceloporus grammicus

Fuente: elaboración propia basada en los datos de González-Morales et al., 2017.

Nota: nótese la relación cuadrática entre el valor hemático y la altitud.

La biología térmica en el grupo Sceloporus, al igual que otros organismos, es conservativa, aunque las temperaturas de selección varían con la altitud, incluso observamos y registramos en el laboratorio individuo activos a los $5{ }^{\circ} \mathrm{C}$ (González-Morales et al., 2019). Está por demás insistir que esta especie puede hacer frente al calentamiento global gracias a su amplio rango de distribución altitudinal. Aparentemente, las lagartijas del género Sceloporus pueden presentar respuesta hemática ante la hipoxia; sin embargo, trabajos en otros grupos de reptiles son escasos o no existen, con la excepción de la lagartija Phrinocephalus vlangalii, que muestra respuestas similares a las observadas en $S$. torquatus (He et al., 2013). Además, en nuestro laboratorio estudiamos a la especie Heloderma horridum con la finalidad de observar si estos organismos son capaces de responder a la hipoxia de manera hemática (Guadarrama et al., 2019).

$H$. horridum es un lagarto venenoso, cuya distribución se registra desde Sonora hasta Chiapas, incluso hasta Guatemala. Su distribución altitudinal se reporta desde el nivel del mar y hasta los $1200 \mathrm{~m}$. Es una especie considerada ante las leyes mexicanas como "amenazada" debido a la destrucción de su hábitat (Guadarrama et al., 2019). Por esta razón su manutención y reproducción en cautiverio es común, aunque lamentablemente muchos zoológicos o herpetarios los mantienen fuera de su rango altitudinal natural (por ejemplo Africam Zafari en Puebla, a $2400 \mathrm{~m})$.

Como se puede observar en la gráfica 5 y figura 3, H. horridum no muestra cambios en sus valores hemáticos a dos diferentes altitudes, lo cual puede ser explicado, al menos en parte, por la biología de esta especie, de la cual sabemos habita en madrigueras escasamente oxigenadas durante grandes periodos (hasta 62 días) (Ariano-Sánchez y Salazar, 2015). Dicha adaptación es posible gracias a las derivas sanguíneas que ocurren en lagartos (Hicks, 2002). Este fenómeno permite a los lagartos hiperoxigenar la sangre en ambientes hipóxicos. Brevemente, la sangre desoxigenada proveniente del cuerpo pasa a los pulmones para ser oxigenada, de ahí regresa al corazón y, en un ciclo normal, sería enviada al cuerpo para su uso; sin embargo, en las derivas 
sanguíneas de los lagartos la sangre oxigenada de manera parcial de los pulmones es enviada al corazón, de donde es bombeada de nuevo a los pulmones para su hiperoxigenación, lo cual es posible gracias a la división parcial del ventrículo cardiaco de estos animales (Birchard et al., 1984; Hillman et al., 2009; Rios et al., 2013). Ese mecanismo permitiría a $H$. horridum sobrevivir en ambientes hipóxicos, pero su biología térmica poco estudiada nos indica que tiene un rango de temperaturas de actividad que lo limitan a ambientes cálidos, pues va de los 19.9 a los $29.5^{\circ} \mathrm{C}$ (Beck, 2006), por lo que una migración altitudinal es poco probable. Por ello, concluimos que ésta sería una especie altamente vulnerable al calentamiento global.

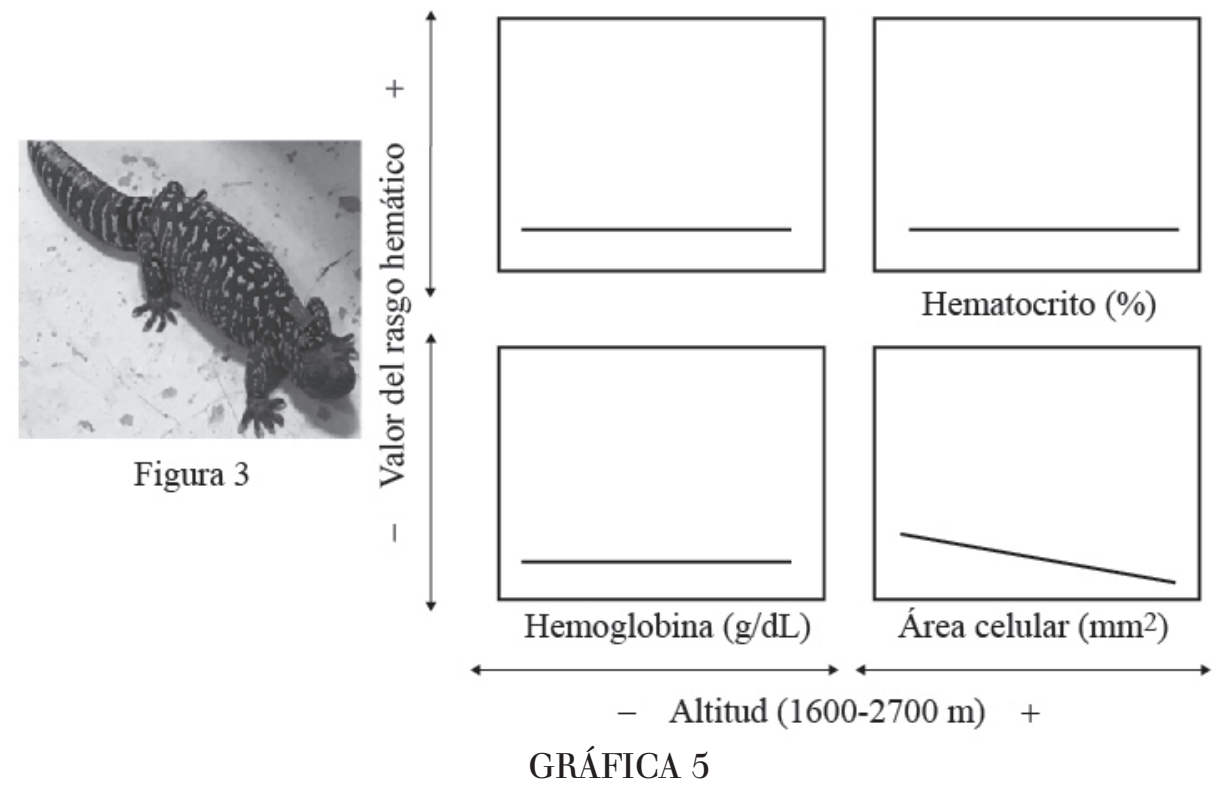

Valores hemáticos observados en Heloderma horridum

Fuente: elaboración propia basada en los datos de Guadarrama et al., 2019.

Nota: nótese que no existe relación entre los valores y la altitud.

\section{Prospectiva}

Ante el incremento de la temperatura ambiental, parece lógico explorar la idea de la posible migración altitudinal de las especies ectotermas. Esta hipótesis está basada en la biología térmica de muchos saurios; sin embargo, como ya hemos discutido aquí, la hipoxia es otro factor que debemos tomar en cuenta para poner a prueba esta hipótesis (González-Morales, 2015, 2017; Guadarrama et al., 2019), además de la fragmentación del hábitat cada vez más frecuente (González-Morales, 2015, 2017).

Es necesario entonces entender si la respuesta hemática está presente en otros grupos de reptiles o anfibios. Nuestro laboratorio también ha demostrado que hay cambios morfológicos en los sistemas de intercambio gaseoso en anfibios del género Ambystoma en relación con la altitud (Arredondo et al., 2017). No obstante, se necesitan realizar estudios de cambios morfológicos en los sistemas cardiovasculares o en el metabolismo de lagartijas de alta o baja montaña, lo que nos permitiría conocer si hay otros mecanismos involucrados con los cuales los ectotermos puedan afrontar el calentamiento global como cambios en el tamaño del corazón y de los pulmones o incluso de la microcapilaridad a nivel de tejidos. Otros aspectos metabólicos también son importantes: cambios en las fibras musculares, donde individuos en ambientes hipóxicos muestren predominancia de fibras anaerobias lo que demandaría menor cantidad de oxígeno y de glucosa para sus actividades diarias y que se vería reflejado en la disminución del estrés oxidativo y por ende en una mayor longevidad. Todas estas son peguntas aún sin explorarse en los diversos grupos de reptiles. 


\section{Agradecimientos}

Queremos agradecer a la Secretaría de Investigación y Estudios Avanzados de la Universidad Autónoma del Estado de México por su el apoyo económico para la realización de estos estudios, así como al Consejo Nacional de Ciencia y Tecnología, a CEPANAF y a los revisores anónimos que ayudaron a mejorar este escrito.

\section{REFERENCIAS}

Adolph, S. C., \& Porter, W. P. (1993). Temperature, activity, and lizard life histories. American Naturalist, 142, 273-295.

Andrews, R. M. (1998). Geographic variation in field body temperature of Sceloporus lizards. Journal of Thermal Biology, 23, 329-334.

Ariano-Sánchez, D., \& Salazar, G. (2015). Spatial ecology of the endangered Guatemalan beaded lizard Heloderma charlesbogerti (Sauria: Helodermatidae), in a tropical dry forest of the Motagua Valley, Guatemala. Mesoamerican Herpetology, 2(1), 64-74.

Arredondo, J., González-Morales, J. C., Rodríguez-Antolín, J., Bastiaans, E., Monroy-Vilchis, O., Manjarrez, J., \& Fajardo, V. (2017). Histological Characteristics of gills and dorsal skin in Ambystoma leorae and Ambystoma rivulare: Morphological changes for living at high altitude. International Journal of Morphology, 35(4), 1590-1596.

Barry, R. G., \& Chorley, R. J. (2003). Atmosphere, weather, and climate. New York: Routledge Taylor \& Francis Group,.

Beck, D. (2006). Biology of Gila monster and beaded lizards. California: University of California Press.

Birchard, G. F., Kilgore, D. L., \& Bogss, D. F. (1984). Respiratory gas concentrations and temperatures within the burrows of three species of burrow-nesting birds. The Wilson Bulletin, 96, 451-456

Bouverot, P. (1985). Adaptation to altitude-hypoxia in Vertebrates. Berlin: Springer-Verlag.

Brohan, P., Kennedy, J. J., Harris, I., Tett, S. F. B., \& Jones P. D. (2006). Uncertainty estimates in regional and global observed temperature changes: A new dataset from 1850. Journal de Geophysical Research, 111, D12106.

Caballero, M., Lozano, S. y Ortega, B. (2007). Efecto invernadero, calentamiento global y cambio climático: una perspectiva desde las ciencias de la tierra. Revista Digital Universitaria, 8(10), 2-12.

Crowley, S. R. (1985). Thermal sensitivity of sprint-running in the lizard Sceloporus undulatus: Support for a conservative view of termal physiology. Oecologia, 66, 219-225.

Feria-Ortíz, M., Nieto-Montes de Oca, A., \& Salgado-Ugarte, I. (2001). Diet and reproductive biology of the viviparus lizard Sceloporus torquatus torquatus (Squamata: Phrynosomatidae). Journal of Herpetology, 35(1), 104-112

Flores-Villela, O. A., \& Gerez, P. (1994). Biodiversidad y conservación en México: vertebrados, vegetación y uso de suelo. México: CONABIO-UNAM.

González-Morales, J. C., Quintana, E., Díaz-Albiter, E. H., Guevara-Fiore, P., \& Fajardo, V. (2015). Is the erythrocyte size a strategy to avoid hypoxia in Wiegmann's Torquate Lizards (Sceloporus torquatus)? Field evidence. Canadian Journal of Zoology, 93, 377-382.

González-Morales, J. C., Beamonte-Barrientos, R., Bastiaans, E., Guevara-Fiore, P., Quintana, E., \& Fajardo, V. (2017). A mountain or a plateau? Hematological traits vary nonlinearly with altitude in Highland lizard. Physiological and Biochemical Zoology, 90(6), 638-645. 
González-Morales, J. C., Rivera-Rea, J., Moreno-Rueda, G., Bastiaans, E., Díaz de la Vega-Pérez, A. H., Bautista-Ortega, A., \& Fajardo, V. (2020). To be small and dark is advantageous for gaining heat in mezquite lizards, Sceloporus grammicus (Squamata: Phrynosomatidae). Biological Journal of the Linnean Society. In press.

Guadarrama, S., Domínguez-Vera, H., Díaz-Albiter, H. M., Quijano, A., ... Bastiaans E. (2020). Hypoxia by altitude and welfare of captive Beaded lizards (Heloderma horridum) in Mexico: Hematological approaches. Journal of Applied Animal Welfare Science, 23(1), 74-83.

He, J., Xiu, M., Tang, X., Yue, F., Wang, N., Yang, S., \& Chen, Q. (2013). The different mechanisms of hypoxic acclimatization and adaptation in lizard Phrynocephalus vlangalii living on Qinghai-Tibet Plateau.Journal of Experimental Zoology Part A: Ecological Genetics and Physiology, 319(3), 117-123.

Hicks, J. W. (2002). The physiological and evolutionary significance of cardiovascular shunting patterns in reptiles. American Journal of Physiology, 17, 241-245.

Hillman, S. S. (2009). Ecological and environmental physiology of amphibians. New York: Oxford University Press.

IPCC (Intergovernmental Panel on Climate Change). (2018). Summary for Policymakers of IPCC Special Report on Global Warming of $1.5^{\circ} \mathrm{C}$ approved by governments. IPCC.

Lemos-Espinal, J. A., \& Ballinger R. E. (1995). Comparative termal ecology of the high-altitude lizard Sceloporus grammicus on the eastern slope of the Iztaccihuatl Volcano, Puebla, Mexico. Canadian Journal of Zoology, 73, 2184-2191.

Méndez de la Cruz, F. R. y Gutiérrez-Mayén, M. G. (1991). Variación de la robustez física de Sceloporus torquatus (Sauria: Iguanidae) y sus implicaciones sobre la temporada de reproducción. Acta Zoológica Mexicana, $46,1-12$

Ortega, Z., Mencia, A., \& Pérez-Mellado, V. (2016). Adaptative seasonal shifts in the termal preferences of the lizard Iberolacerta galani (Squamata: Lacertidae). Journal of Thermal Biology, 62, 1-6.

Petit, J. R., Jouzel, J., Raynaud, D., Barkov, N. I., ... Barnola, J. M. (1999). Climate and atmospheric history of the past 420,000 years from the Vostok ice core, Antarctica. Nature, 399, 429-436.

Pough, F. H. (1980). The adventages of ectothermy for tetrapods. Am. Nat., 115, 92-112.

Pough, F. H., Andrews, R. M., Cadle, J. E., Crump, M. L., ...Savitzky, A. H. (2001). Herpetology. New York: Prentice Hall.

Rios, R. L., Rodríguez de, R. F. J., Velázquez, R. A. S., \& Hernández, F. A. A. (2013). Morfometría geométrica del corazón de Hyla plicata a través de un gradiente altitudinal en el eje Neovolcánico Mexicano. The International Journal of Morphology, 3, 905-910.

Rivera-Rea, J., González-Morales, J. C., Bastiaans, E., \& Fajardo, V. (2018). SCELOPORUS TORQUATUS (Torquate Lizard). Selected body temperature. Herpetological Review, 49(3).

Ruiz, G., Rosenmann, M., \& Veloso, A. (1983). Respiratory and hematological adaptation to high altitude in Telmantobius frog from Chilean Andes. Comparative Biochemistry and Physiology A, 76, 109-114.

Ruiz, G., Rosenmann, M., \& Veloso, A. (1989). Altitudinal distribution and blood values in the toad, Bufo spinulosus Wiegmann. Comparative Biochemistry and Physiology, 94(4), 643-646.

Sinervo, B., Méndez- de la Cruz, F. R., Miles, D. B., Heulin, B., ... Bastiaans, E. (2010). Erosion of lizard diversity by climate change and altered niches. Science, 328, 894-899.

Sinervo, B., Miles, D. B., Wu, Y., Méndez-de la Cruz, F. R., ... Kirchhofet, S. (2018). Climate change, thermal niches, extinction risk and maternal-effect rescue of toad-headed lizards, Phrynocephalus, in thermal extremes of the Arabian Peninsula to the Qinghai-Tibetan Plateau. Integrative Zoology, 13, 450-470 
Snyder, G. K., \& Weathers, W. W. (1997) Activity and oxygen consumption during hypoxic exposure in high altitude and lowland sceloporine lizards. Journal of Comparative Physiology B, 117, 291-301.

Storz, J. F., \& Moriyama, H. (2008) Mechanims of hemoglobin adaptation to high altitude hypoxia. High Altitude Medicine \& Biology, 9(2), 148-157.

Uribe-Peña, Z. A., Ramírez-Bautista, A., \& Casas-Andreu, G. (1999). Anfibios y reptiles de las serranias del Distrito Federal, México. UNAM: Instituto de Biología.

Wheathers, W., \& White, W. (1972). Hematological observations on populations of the lizard Sceloporus occidentalis from sea level and altitude. Herpetologica, 28(2), 172-175.

\section{BY-NC-ND}

